\title{
Muerte por ahorcadura en necropsias de ley
}

\author{
Death by hanging at \\ necropsy law
}

\section{Ulises P.Mejia Rodriguez ${ }^{1}$ Alex R. Mejía Rodriguez ${ }^{2}$}

1. Médico Legista, Master en Medicina Forense, Master en Gerencia Social, Doctorando en Criminalística. Instituto de Medicina Legal Perú.

2. Medico Cirujano en Essalud Tacna, U.N.J.B.G. - Tacna

\section{RESUMEN}

OBJETIVO: Determinar las lesiones macro y microscopicas y sus caracteristicas en las muertes por ahorcadura. MATERIAL Y MÉTODOS: Se realizo un estudio descriptivo prospectivo de enero 2010 a diciembre del 2015. RESULTADOS:De 39 casos, el $84,62 \%$ (33/39) varones, el mayor porcentaje descansa sobre los cadaveres necropsiados entre 13 a 17 años con 48,72\% (19/39), la media de edad fue 21 años, La provincia de procedencia más frecuente fue Chucuito con 46,15\% (18/39), 43,59\% (17/39) fueron estudiantes, la escena del hecho correspondió al area rural 84,62\% (33/39); el 100\% tuvo etiología médico legal sucida. Se encontró lesiones comunes al sindrome asfictico general como son la coloracion oscura, hiperfluides de la sangre, congestion de organos en el 100\% (39/39) de los casos. Se encontró en los ahorcados 2 tipos de fascies, el anemico con $15,39 \%(6 / 39)$ y el congestivo $84,62 \%$ (33/39); en la region cervical en el $100 \%$ (39/39) de los casos coloracion violacea de los bordes del surco (Signo de Thoinot), livideces en placas por encima y por debajo del surco(Signo de Ponsold), impresion de la trama del lazo en el fondo del surco(Signo de Bonnet). CONCLUSIONES:Las lesiones por ahorcamiento plantean dificultades al establecer el diagnóstico de muerte, por lo tanto hay que hacer un debido procedimiento.

PALABRAS CLAVE: Muerte por ahorcamiento, Asfixia, necropsia de ley, lesiones microscopicas, lesiones macroscopicas.

\section{ABSTRACT}

OBJECTIVE: To determine the mechanical asphyxia by hanging in deaths or suspension, macro and microscopic lesions and their characteristics. MATERIAL AND METHODS: A prospective study was conducted from January 2010 to December 2015. RESULTS: Of 39 cases, $84.62 \%$ (33/39) males, the highest percentage rests on the bodies autopsied between 13 to 17 years with $48.72 \%$ (19/39), the mean age was 21 years, the province most common source was Chucuito with $46.15 \%$ (18/39), $43.59 \%$ (17/39) were students, the scene made rural area corresponded to $84.62 \%$ (33/39); $100 \%$ had sucida legal medical etiology. common injuries to the general asphyxia syndrome was found like dark coloration, hiperfluides blood, organ congestion in 100\% (39/39) of cases. 2 types of facies, the anemic with $15.39 \%$ $(6 / 39)$ and $84.62 \%$ congestive (33/39) was found hanged; in the cervical region in $100 \%$ (39/39) of cases violaceous edges of the groove (sign Thoinot), livideces plates above and below the groove (sign Ponsold), printing weft loop on the bottom of the groove (Bonnet sign). CONCLUSIONES: hanging injuries pose difficulties in establishing the diagnosis of death, therefore we must make a due process.

KEYWORDS: Death by hanging, asphyxia, law necropsy, microscopic lesions, gross lesions. 


\section{INTRODUCCIÓN}

En el Perú, es frecuente los suicidios, es asi que la muerte por ahorcadura es un diagnostico de muerte suicida de tipo violenta. Es sabido que la modalidad de suicidio está muy ligada a factores culturales y al sexo, existiendo grandes diferencias de unas regiones a otras. La muerte por ahorcadura, es una asfixia mecanica, que se producen por acción del peso del propio peso del cuerpo que ejerce una compresion extrinseca del cuello. Cuando la presión sobre el cuello se ejerce por una fuerza distinta del peso del cuerpo la asfixia se denomina estrangulación.

Las ahorcaduras se clasifican según su resultado en Consumada o mortal y Tentativa o semi-ahorcamiento. Si bien el desarrollo del tema se centra en el trabajo médico-forense con el cadáver, no se debe pasar por alto que son frecuentes los casos de ahorcamiento no consumado (principalmente: intento de suicidio, accidente en la infancia). Según su etiología médicolegal es Intencional que puede ser Suicida u Homicida; Accidental que se sub divide en Accidental propiamente dicha, Lúdica, Erótica. Como un dato de interés histórico se podría agregar la ahorcadura judicial, como método de pena de muerte. La etiología médico-legal suicida es ampliamente preponderante. Según la existencia de contacto del cuerpo con el suelo puede ser Completa o Incompleta. No es necesaria la fuerza de todo el peso del cuerpo para provocar rápidamente la muerte por ahorcamiento. Según la disposición del lazo: Simétrico y asiemtrico; La forma más frecuente es la asimétrica con el nudo lateralizado, ubicado por debajo del ángulo del maxilar inferior. Asimismo, pueden clasificarse en simétricas y asimétricas, dependiendo de la posición donde se coloque el nudo; en las simétricas el nudo está situado en la línea media del cuello, distinguiéndose entre ahorcadura simétrica anterior y posterior. También pueden clasificarse como ahorcaduras típicas y atípicas; las típicas se corresponden con aquellas en las que el nudo ocupa la línea media posterior del cuello. Dependiendo de esta clasificación podemos encontrar distintos cuadros lesiónales.

Se han descritos varios mecanismos de muerte: anoxia anóxica, isquemia encefálica, inhibición refleja y lesión medular.En la anoxia anóxica, el lazo se va hacia la parte superior del cuello y provoca una retropulsión del hioides y de la base de la lengua, que se adosa a la cara posterior de la faringe bloqueando el paso del aire.

Su etiología médico legal en la mayoría de casos es suicida; predomina en varones y en estudiantes(2). Esta entidad produce en los cadáveres lesiones y signos externos, como son los signos generales de asfixia y otros hallasgos encontrados a nivel del cuello.

El objetivo del presente trabajo es determinar las causas de muerte por ahorcamiento y sus caracteristicas. Asimismo, es importante recordar que la necropsia de un cadáver debe de ser completa, metódica, descriptiva y científica. Será sustentada con todos los medios disponibles para llegar a ello y así contribuir como auxiliares para una buena impartición de justicia.

\section{MATERIAL Y MÉTODOS}

Estudio descriptivo prospectivo realizado en tres Divisiónes Médico Legales (DML) que son la DML I Chucuto Juli, DML I El Collao llave y la DML I Yumguyo ubicadas en al sur de la ciudad de Puno durante los meses de Enero 2010 a Diciembre 2015. Para el estudio se selecciono las necropsias medico legales por muertes por ahorcamiento, las que fueron solicitadas por la Fiscalia provincial Penal de la provincia a la que pertenecen cada una de estas DML.

Se procedio a las tomas fotograficas de acuerdo a la normatividad vigente, al manual de Tanatologia Forense del Instituto de Medicina Legal del Perú; siendo asi y amparandonos en las consideraciones del acto medico y en el sigilio pericial omitimos la identificaciòn de los cadaveres.

La población de estudio son todos los cadaveres fallecidos por Ahorcamiento (Asfixia mecanica por ahorcamiento o suspencion) que fueron necropsiados en la DML I Chucuto Juli, DML I El Collao llave y la DML I Yunguyo respectivamente.Se determinó y describió las lesiones y caracteristicas del cadaver a nivel del cuello, y en otras localizaciones, asi como los signos generales de asfixia.

Las necropsisas de ley, las realizamos en presencia del Representante del Ministerio Publico (Fiscal Penal de Turno), personal de la Policia Nacional del Perú, segun lo normado en el Manual de Procedimientos Tanatologicos Forenses del Instituto de Medicina Legal y Ciencias Forenses del Perú.

No se excluyo del estudio a ningun cadaver, ya que a los 39 cadaveres se les realizo la necropsia de ley. Los datos fueron procesados en el programa Microsoft Exel 2013. 


\section{RESULTADOS}

En el estudio realizado durante los meses de enero 2010 a diciembre 2015 en lasDML I Chucuto Juli, DML I El Collao llave y la DML I Yunguyo respectivamente, se realizaron 39 Necropsias de Ley(NEC), en las que se determino como causa de muerte la muerte por Ahorcamiento y como agente causante unagente constrictor flexible al cuello (lazo).(Tabla 1), (Figura 1).

\begin{tabular}{|c|c|c|c|c|c|c|c|c|}
\hline \multicolumn{9}{|c|}{ MUERTE POR AHORCAMIENTO SEGÚN GRUPO ETAREO } \\
\hline & \multirow{2}{*}{\multicolumn{2}{|c|}{$\begin{array}{l}\text { DML I CHUCUITO } \\
\text { JULI }\end{array}$}} & \multirow{2}{*}{\multicolumn{3}{|c|}{$\begin{array}{l}\text { DMLI IEL COLLAO } \\
\text { ILAVE }\end{array}$}} & \multirow{2}{*}{$\begin{array}{c}\text { DMLI } \\
\text { YUNGUYO }\end{array}$} & \multicolumn{2}{|c|}{ TOTAL } \\
\hline & & & & & & & $\mathrm{N}$ & $\%$ \\
\hline -11 Meses & 0 & 0.00 & 0 & 0.00 & 0 & 0.00 & 0 & 0.00 \\
\hline 1-12 Años & 2 & 5.13 & 3 & 7.69 & 0 & 0.00 & 5 & 12.82 \\
\hline 13-17 Años & 9 & 23.08 & 7 & 17.95 & 3 & 7.69 & 19 & 48.72 \\
\hline 18-60 Años & 5 & 12.82 & 2 & 5.13 & 5 & 12.82 & 12 & 30.77 \\
\hline Más de 60 Años & 2 & 5.13 & 1 & 2.56 & 0 & 0.00 & 3 & 7.69 \\
\hline TOTAL & 18 & 46.15 & 13 & 33.33 & 8 & 20.51 & 39 & 100.00 \\
\hline
\end{tabular}

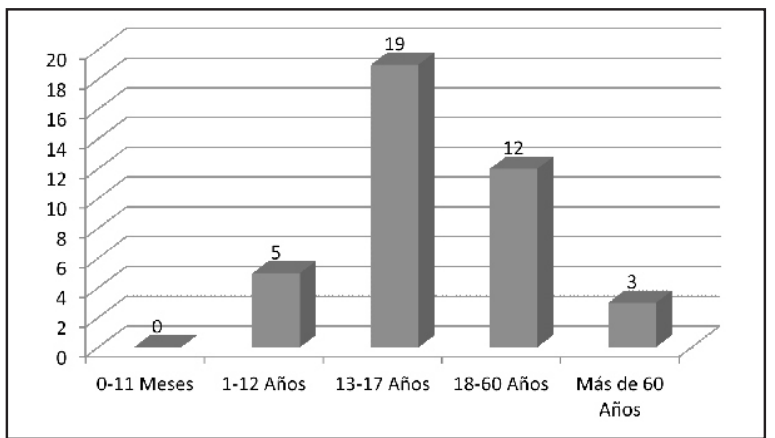

Figura 1. Poblacion de estudio según la edad; cadaveres necropsiados por muerte por Ahorcamiento.

Dentro de la poblacion en estudio, el mayor porcentaje descansa sobre los cadaveres necropsiados entre 13 a 17 años con 48,72\%(19/39), seguido de cadaveres entre 18 a 60 años de edad con 30,77\%(12/39); quienes fueron necropsiados en la Divisiones Medico Legales por muerte por Ahorcamiento(Figura 1).

De las 39 Necropsias de Ley para determinar la muerte por Ahorcamiento, evidenciamos que el $84,62 \%(33 / 39)$ eran de sexo masculino, y el $15,38 \%$ (6/39) fue del sexo femenino. (Tabla 2).

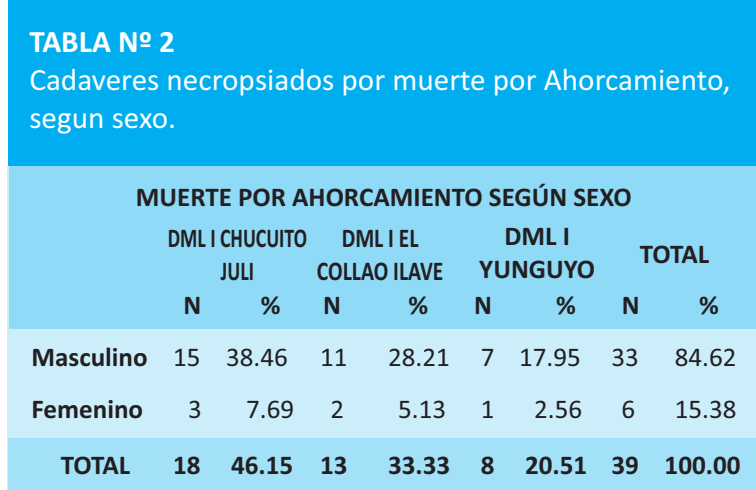

Siendo asi, clasificamos a los cadaveres que se les realizo la necropsia de ley por muerte por ahorcamiento, observandose una mayor frecuencia entre los rangos de edades entre 13 a 17 años con $48,72 \%$ (19/39), seguido de cadaveres entre 18 a 60 años de edad con $30,77 \%$ (12/39); quienes fueron necropsiados en la Divisiones Medico Legales por muerte por Ahorcamiento (Tabla 1).

\section{TABLA № 3 \\ Cadaveres necropsiados por muerte por Ahorcamiento, segun ocupacion del cadaver.}

\begin{tabular}{|c|c|c|c|c|c|c|c|c|}
\hline \multicolumn{9}{|c|}{ MUERTE POR AHORCAMIENTO SEGÚN OCUPACION } \\
\hline & \multicolumn{2}{|c|}{$\begin{array}{c}\text { DMLI CHUCUITO } \\
\text { JULI }\end{array}$} & \multicolumn{2}{|c|}{$\begin{array}{c}\text { DML I EL } \\
\text { COLLAO ILAVE }\end{array}$} & \multicolumn{2}{|c|}{$\begin{array}{c}\text { DMLI } \\
\text { YUNGUYO }\end{array}$} & \multicolumn{2}{|c|}{ TOTAL } \\
\hline & $\mathbf{N}$ & $\%$ & $\mathbf{N}$ & $\%$ & $\mathbf{N}$ & $\%$ & $\mathbf{N}$ & $\%$ \\
\hline Agricultor & 3 & 7.69 & 4 & 10.26 & 1 & 2.56 & 8 & 20.51 \\
\hline Ganadero & 4 & 10.26 & 3 & 7.69 & 1 & 2.56 & 8 & 20.51 \\
\hline Profesor & 1 & 2.56 & 1 & 2.56 & 0 & 0.00 & 2 & 5.13 \\
\hline Estudiante & 9 & 23.08 & 5 & 12.82 & 3 & 7.69 & 17 & 43.59 \\
\hline Obrero & 1 & 2.56 & 0 & 0.00 & 1 & 2.56 & 2 & 5.13 \\
\hline Otros & 0 & 0.00 & 0 & 0.00 & 2 & 5.13 & 2 & 5.13 \\
\hline TOTAL & 18 & 46.15 & 13 & 33.33 & 8 & 20.51 & 39 & 100.00 \\
\hline
\end{tabular}

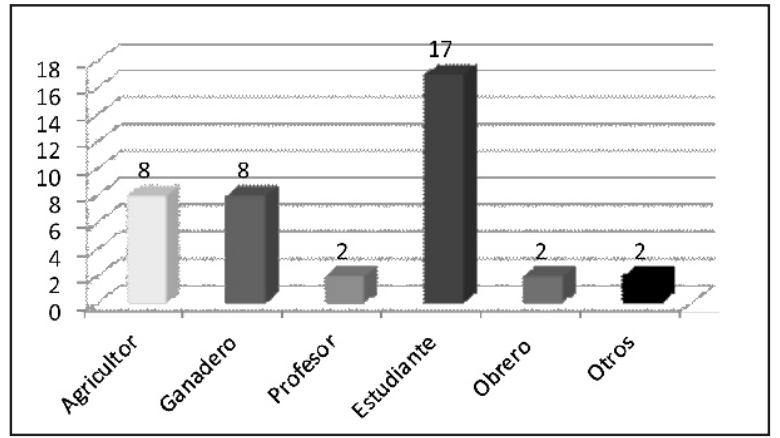

La media de edad fue 21 años (rango de 01 a mas de 60 años); $43,59 \%$ (17/39) fueron estudiantes y $41,02 \%$ (16/39) fueron ganaderos y agricultores (Tabla 3). El año en el que se presentó mayor cantidad de muertes 
por ahorcamiento fue en el año 2010con 20,51\% (8/39). La provincia de procedencia más frecuente fueChucuito con46,15\% (18/39), seguido de El Collao Ilave con 33,33\% (13/39).

\section{TABLA № 4 \\ Cadaveres necropsiados por muerte por Ahorcamiento, segun año en el que se ahorcaron y de acuerdo a las DML donde se necropcio el cadaver.

\begin{tabular}{|c|c|c|c|c|c|c|c|c|}
\hline \multicolumn{9}{|c|}{$\begin{array}{l}\text { MUERTE POR AHORCAMIENTO SEGÚN DIVIS } \\
\text { LEGAL DE PROCEDENCIA }\end{array}$} \\
\hline & \multicolumn{2}{|c|}{$\begin{array}{l}\text { DMLI I CHUCUITO } \\
\text { JULI }\end{array}$} & \multicolumn{2}{|c|}{$\begin{array}{l}\text { DML IEL } \\
\text { COLLAO ILAVE }\end{array}$} & \multicolumn{2}{|c|}{$\begin{array}{l}\text { DML } \\
\text { YUNGUYO }\end{array}$} & \multicolumn{2}{|c|}{ TOTAL } \\
\hline & $\mathbf{N}$ & $\%$ & $\mathrm{~N}$ & $\%$ & $\mathbf{N}$ & $\%$ & $\mathbf{N}$ & $\%$ \\
\hline Año 2010 & 5 & 12.82 & 2 & 5.13 & 1 & 2.56 & 8 & 20.51 \\
\hline Año 2011 & 4 & 10.26 & 1 & 2.56 & 2 & 5.13 & 7 & 17.95 \\
\hline Año 2012 & 3 & 7.69 & 2 & 5.13 & 1 & 2.56 & 6 & 15.38 \\
\hline Año 2013 & 1 & 2.56 & 5 & 12.82 & 1 & 2.56 & 7 & 17.95 \\
\hline Año 2014 & 3 & 7.69 & 2 & 5.13 & 1 & 2.56 & 6 & 15.38 \\
\hline Año 2015 & 2 & 5.13 & 1 & 2.56 & 2 & 5.13 & 5 & 12.82 \\
\hline TOTAL & 18 & 46.15 & 13 & 33.33 & 8 & 20.51 & 39 & 100.00 \\
\hline
\end{tabular}

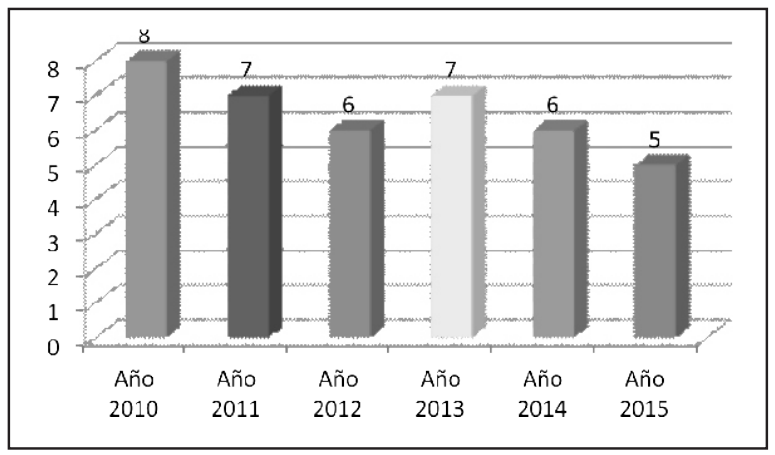

La escena del hecho correspondió al area rural $84,62 \%$ (33/39) y al area urbana $15,38 \%$ (6/39). El 100\% (39/39) tuvo etiología médico legal suicida. (Tabla 5 ).

\section{TABLA № 5}

Cadaveres necropsiados por muerte por Ahorcamiento, segun lugar de ocurrencia.

\begin{tabular}{|c|c|c|c|c|c|c|c|c|}
\hline \multicolumn{9}{|c|}{ MUERTE POR AHORCAMIENTO SEGÚN LUGAR DE OCURRENCIA } \\
\hline & \multicolumn{2}{|c|}{$\begin{array}{c}\text { DMLI CHUCUITO } \\
\text { JULI }\end{array}$} & \multicolumn{2}{|c|}{$\begin{array}{c}\text { DML I EL } \\
\text { COLLAO ILAVE }\end{array}$} & \multicolumn{2}{|c|}{$\begin{array}{c}\text { DML } \\
\text { YUNGUYO }\end{array}$} & \multicolumn{2}{|c|}{ TOTAL } \\
\hline & $\mathbf{N}$ & $\%$ & $\mathbf{N}$ & $\%$ & $\mathrm{~N}$ & $\%$ & $\mathbf{N}$ & $\%$ \\
\hline Area Rural & 15 & 18.46 & 11 & 28.21 & 7 & 17.95 & 33 & 84.62 \\
\hline Area Urbana & 3 & 7.69 & 2 & 5.13 & 1 & 2.56 & 6 & 15.38 \\
\hline TOTAL & 18 & 46.15 & 13 & 33.33 & 8 & 20.51 & 39 & 100.00 \\
\hline
\end{tabular}

En la necropsia médico legal se observo elementos comunes al sindrome asfictico general como son la coloracion oscura, hiperfluides de la sangre, congestion de organos en el 100\% (39/39) de los casos. Se encontró en los ahorcados 2 tipos de fascies, el anemico con $15,39 \%(6 / 39)$ y el congestivo $84,62 \%$ (33/39).

\section{TABLA No 6}

Externos en la Region Cervical en Cadaveres necropsiados por muerte por Ahorcamiento

\begin{tabular}{|c|c|c|c|}
\hline \multirow[b]{2}{*}{ SIGNO } & \multirow{2}{*}{$\begin{array}{l}\text { SIGNOS MACROSCOPICOS EXTERNOS EN REGION CERVICAL } \\
\text { MUERTE POR AHORCAMIENTO } \\
\text { DESCRIPCION }\end{array}$} & \multicolumn{2}{|c|}{ TOTAL } \\
\hline & & N & $\%$ \\
\hline Thoinot & coloración violácea de los bordes del surco & 39 & 100.00 \\
\hline Azevedo Neves & livideces puntiformes por encima y por debajo de los bordes del surco & 36 & 92.31 \\
\hline Ponsold & livideces en placas por encima por debajo del surco & 39 & 100.00 \\
\hline Schulz & borde superior del surco saliente y violáceo & 35 & 89.74 \\
\hline Bonnet & impresión de la trama del lazo en el fondo del surco & 39 & 100.00 \\
\hline Ambroise Paré & erosión de la piel del fondo del surco & 23 & 58.97 \\
\hline Neyding & infiltrado hemorragico petequial del fondo del surco & 15 & 38.46 \\
\hline Lesser & vesículas sanguinolentas en el fondo del surco & 18 & 46.15 \\
\hline
\end{tabular}

Al examen macroscopico externo,en los casos de muerte por ahorcameinto, se observo en la region cervical en el $100 \%$ (39/39) de los casos coloracion violacea de los bordes del surco (Signo de Thoinot), livideces en placas por encima y por debajo del surco(Signo de Ponsold), impresion de la trama del lazo en el fondo del surco(Signo de Bonnet).

Dentro de los signos macroscopicos internos en las partes blandas del cuello, se encontro en el $100 \%$ (39/39) condensacion del tejido celular subcutaneo a nivel del surco, seguido de una infiltracion del usculo esternocleido mastoideo en el 94,87\%(37/39).

\section{TABLA № 7}

Signos Macroscopicos Internos en Partes blandas del cuello en Cadaveres necropsiados por muerte por Ahorcamiento

\begin{tabular}{|c|c|c|c|}
\hline \multicolumn{4}{|c|}{ SIGNOS MACROSCOPICOS INTERNOS EN PARTES BLANDAS DEL CUELLO } \\
\hline \multirow[b]{2}{*}{ SIGNO } & \multirow[t]{2}{*}{ MUERTE POR AHORCAMIENTO } & \multicolumn{2}{|c|}{ TOTAL } \\
\hline & & $\mathrm{N}$ & $\%$ \\
\hline Thoinot-Lacassagne & $\begin{array}{l}\text { Condensación del tejido celular subcutáneo a nivel del } \\
\text { surco (línea argentina) }\end{array}$ & 39 & 100.00 \\
\hline Martin & infiltración del músculo esternocleidomastoideo & 37 & 94.87 \\
\hline Hofmann-Haberda & infiltración de los músculos profundos del cuello & 33 & 84.62 \\
\hline Lesser & $\begin{array}{l}\text { desgarro transversal con infiltración del músculo } \\
\text { tiro-hiodeo }\end{array}$ & 7 & 17.95 \\
\hline
\end{tabular}

De los hallazgos macroscópicos de necropsia, se encontró signos generales de asfixia (cianosis, fluidez sanguínea, hemorragias petequiales en serosas y congestión multivisceral) en todos los casos. 


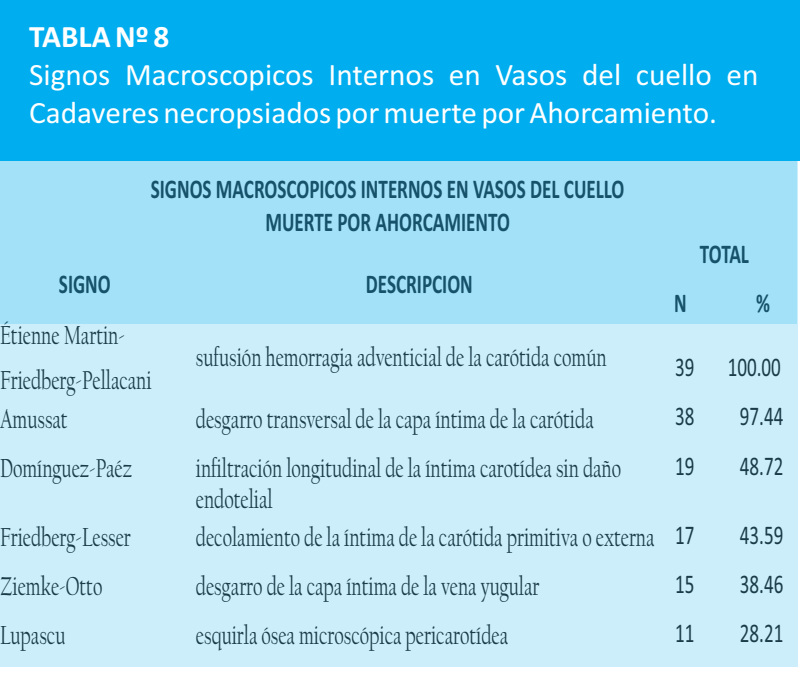

Los hallazgos macroscopicos más frecuentes durante el examen macroscópico interno en los vasos del cuello, en la necropsia médico legal, exceptuando los signos generales de asfixia, fueron: la sufusion hemorragica advenciald e la carotica comun en el 100\% (39/39) de casos, seguido de el desgarro transversa de la capa intima de la carotida en el 97,44\% (38/39) de los casos, asi mismo se observo infiltracion longitudinal de la intima carotidea sin daño endotelial en el $48,72 \%$ (19/39), decolamiento de la intima de la carotida primitiva o externa en el 43,59\% (17/39). (Tabla 7).

Los signos macroscopicos internos en el aparato osteocartilaginoso a nivel del cuello que se presentaron con mayor frecuencia son la fractura de hueso hioides con 84,62\% (33/39) casos. (Tabla 8).

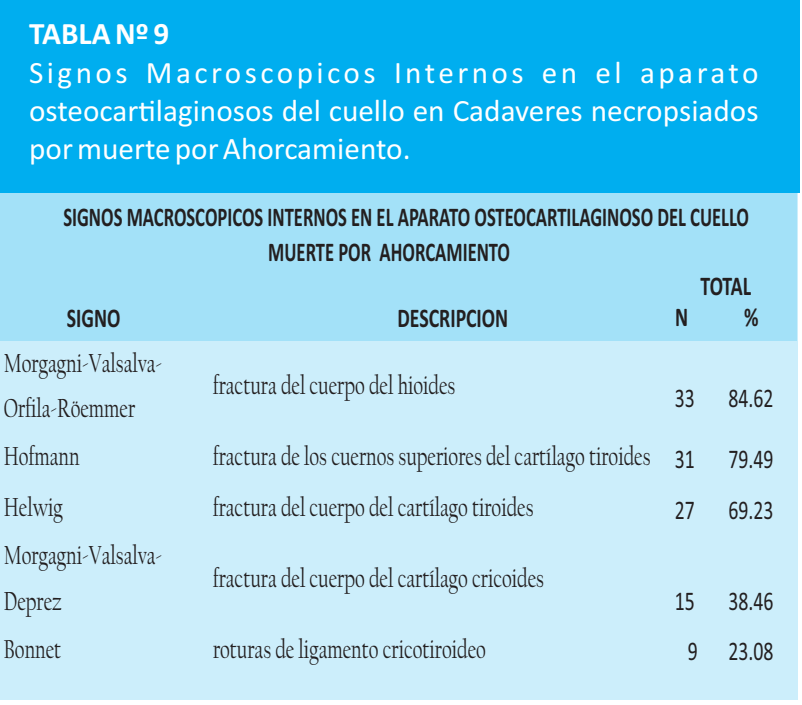

Es raro encontrar signos macroscopicos interno en la columna cervical, es asi que solo encontramos fractura de la apofisis atlantoidea del axis en 10,26\% (4/39) casos y fractura del cuerpo de c1 y c2 en el 2,56\% (1/39) casos.

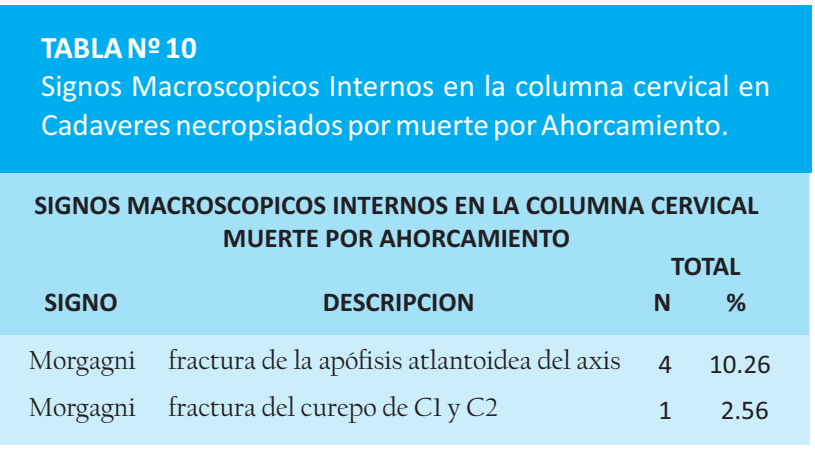

Dentro de los hallazgos macroscopicos internos en la necropsia de ley, al explorar otras estructuras del cuello, se hallo congestion esofagica por encima del surco en $53,85 \%$ (21/39) de casos, seguido de equimosis retrofaringea en $48,72 \%$ (19/39) casos.

\section{TABLA № 11}

Signos Macroscopicos Internos en otras estructuras del cuello en Cadaveres necropsiados por muerte por Ahorcamiento.

\begin{tabular}{|c|c|c|c|}
\hline \multirow[b]{2}{*}{ SIGNO } & \multirow{2}{*}{$\begin{array}{c}\text { MUERTE POR AHORCAMIENTO } \\
\text { DESCRIPCION }\end{array}$} & \multicolumn{2}{|c|}{ TOTAL } \\
\hline & & $\mathbf{N}$ & $\%$ \\
\hline Dotto & $\begin{array}{l}\text { rotura de la vaina de mielina del nervio } \\
\text { neumogástrico }\end{array}$ & 7 & 17.95 \\
\hline $\begin{array}{l}\text { Brouardel-Vibert } \\
\text { Descoust }\end{array}$ & equimosis retrofaríngea & 19 & 48.72 \\
\hline Vargas Alvarado & congestión esofágica por encima del surco & 21 & 53.85 \\
\hline Bonnet & rotura de las cuerdas vocales & 3 & 7.69 \\
\hline
\end{tabular}

En las necropsias de ley realizadas por muerte por ahorcamiento, se pudo evidenciar signos macroscopicos internos en otras localizaciones como son midriasis con miosis contraateral en el $30,77 \%$ (12/39), ademas de infiltracion hemorragica de los discos intervertebrales en la columna lumbar en $23,08 \%$ (9/39) casos.

TABLA No 12
Signos Macroscopicos Internos en otras localizaciones en
Cadaveres necropsiados por muerte por Ahorcamiento.
SIGNOS MACROSCOPICOS INTERNOS EN OTRAS LOCALIZACIONES
MUERTE POR AHORCAMIENTO


Respecto a la alcoholemia en casos de muertes por ahorcamiento, tenemos que en la mayoria de los cadaveres se evidencio contenido de alcohol en la sangre en un $84,62 \%$ (33/39) casos, con cifras comprendidas entre $0,7 \mathrm{~g} \%$ y $1,8 \mathrm{~g} \%$. Respecto a la determinación de sustancias tóxicas, todos los resultados fueron negativos. (Tabla 8 ).

\section{TABLA No 13}

Cadaveres necropsiados por muerte por Ahorcamiento, segun su dosaje etilico.

\begin{tabular}{|c|c|c|c|c|c|c|c|c|}
\hline \multicolumn{9}{|c|}{ MUERTE POR AHORCAMIENTO SEGÚN DOSAJE ETILICO } \\
\hline & \multicolumn{2}{|c|}{$\begin{array}{c}\text { DML I CHUCUITO } \\
\text { JULI }\end{array}$} & \multicolumn{2}{|c|}{$\begin{array}{l}\text { DMLI EL COLLAO } \\
\text { ILAVE }\end{array}$} & \multicolumn{2}{|c|}{ DMLIYUNGUYO } & \multicolumn{2}{|c|}{ TOTAL } \\
\hline & $\mathbf{N}$ & $\%$ & $\mathbf{N}$ & $\%$ & $\mathbf{N}$ & $\%$ & $\mathbf{N}$ & $\%$ \\
\hline Dosaje Etilico (+) & 16 & 41.03 & 12 & 30.77 & 5 & 12.82 & 33 & 84.62 \\
\hline Dosaje Etilico (-) & 2 & 5.13 & 1 & 2.56 & 3 & 7.69 & 6 & 15.38 \\
\hline TOTAL & 18 & 46.15 & 13 & 33.33 & 8 & 20.51 & 39 & 100.00 \\
\hline
\end{tabular}

De los resultados presentados, se aprecia que en todos los casos se halló lesiones o signos generales de asfixia, ademas de otros signos macroscoicos que se han detallado.

\section{DISCUSIÓN}

La muerte por ahorcamiento pertenece al grupo de muertes violentas por suicidio. En nuestro medio se presenta con frecuencia, por ser Puno una ciudad alto andina, con un estrato socio economico medio bajo; sin embargo, es una entidad que preocupa durante las necropsias médico legales por la información que se obtiene de los informes de levantamiento de cadáver. Por ello es necesario conocer sus principales hallazgos macroscópicos internos y externos;ambos estudios complementarios y sus resultados correlacionarse de manera positiva para apoyar el diagnóstico de causa de muerte, sobre todo en casos en los que las lesiones macroscópicas no son evidentes o visibles, certeras o acarreen dudas. Se ha descrito mucho acerca de esta entidad y sus aspectos histopatológicos, tanto en sus lesiones macroscópicas $(1,3,4)$ como microscópicas $(3,5,6)$. En nuestro medio no se cuenta con estudios de esta entidad.

En 2003, Nkolic y col., consideraron que entre el 65\% y $67 \%$ de los ahorcamiento presentan alguna fractura, lo cual concuerda con nuestro estudio, donde se evidencio a nivel del cuello que se presentaron con mayor frecuencia son la fractura de hueso hioides con $84,62 \%$ (33/39) casos, solo encontramos fractura de la apofisis atlantoidea del axis en 10,26\% (4/39) casos, dentro de los hallazgos macroscopicos internos en la necropsia de ley, al explorar otras estructuras del cuello, se hallo congestion esofagica por encima del surco en $53,85 \%$ (21/39) de casos, seguido de equimosis retrofaringea en $48,72 \%$ (19/39) casos.

Actualmente, profesionales medicos experimentan un temor cuando no están capacitados en identificar lesiones en la necropsia de ley y plantear erróneamente el diagnóstico de muerte con el fin de evitar problemas futuros con implicancias médico legales.

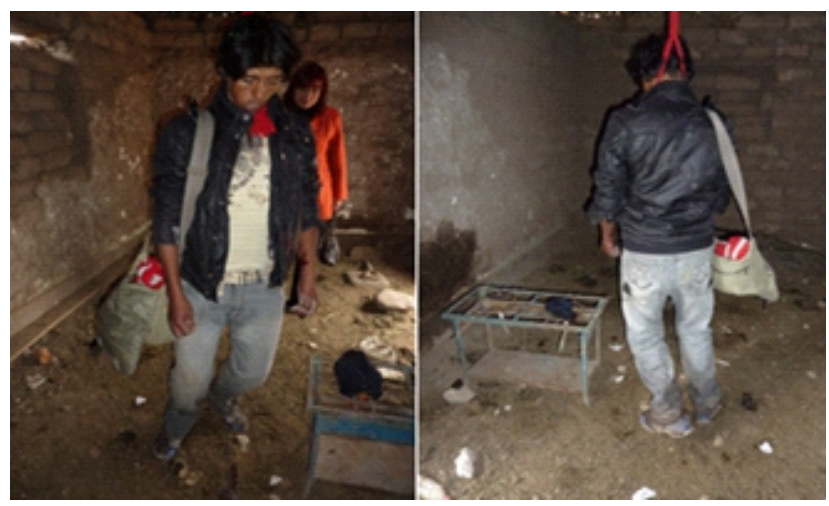

Foto 1. En el levantamiento de cadáver: se observa al cadáver suspendido sobre una cuerda de color rojo (chalina de lana), el cual se encuentra amarrado a una de las vigas del techo donde se encuentra el nudo distal.

Se concluye que las Necropsias de ley son muy útiles y determinantes; por tanto se tiene que tener mucho cuidado ya que la Necropsia de Ley debe de guardar relación de asociación entre lo descrito en el Informe de Levantamiento de cadaver (data y hasllazgos macroscopicos y microscopicos) con las conclusiones emitidas. 


\section{REFERENCIAS BIBLIOGRÁFICAS}

1. Bonnet EPF, Medicina Legal y Toxicología. Buenos Aires: Libreros, 1980.

2. Calabuig G, Villanueva E. Lesiones. Medicina Legal y Toxicología. 5a Ed. Masson, Barcelona, 1998.

3. Código de Procedimientos Penales. Ley 4019. Art. 161. Nombramiento de Peritos. Preferencia y honorarios. Lima, Perú (23 de noviembre del 1939).

4. Código Penal. Decreto Legislativo N.o 635. Art. 121-122. Delitos contra la vida, el cuerpo y la salud. Lesiones.;Perú, 1991.

5. Chalk R, King P. Assesing family violence interventions. Am J PrevMed 1998; 14(4):289-292.

6. Diario Oficial de la Federación. Norma Oficial Mexicana NOM-190-SSA1-1999. Prestación de servicios de salud. Criterios para la atención médica de la violencia familiar. México, DF: Secretaría de Salud; 2000.
7. Di Maio JM, Dana SE Homicidios relacionados con el sexo. En: Manual de patología forense. Ed. Díaz Santos, Madrid, 2003: 165- 167

8. Gayet J, Manual de la Policía Científica. España: Zeus, 1965.

9. Glasser D, Stephen F. Abuso sexual en niños. Ed. Paidós, Buenos Aires, 1997.

10. Gómez de Terreros I: Los profesionales de la salud ante el maltrato. 2ªed. Editorial Comares. Granada, 1997.

11. Guzmán C. Manual de Criminalística, Ediciones LA ROCA, 1a edición, Buenos Aires-Argentina, 2000, p. 487.

12. Gómez de Terreros I: Los profesionales de la salud ante el maltrato. 2aed. Editorial Comares.Granada, 1997.

\section{Correspondencia}

Ulises Papillón Mejía Rodriguez

ulisesmejiarodriguez@hotmail.com
Fecha de recepción: 05 de octubre de 2017

Fecha de aceptación: 20 de noviembre de 2017 